\title{
BMJ Open Diagnostic accuracy study of three alcohol breathalysers marketed for sale to the public
}

\author{
Helen F Ashdown, Susannah Fleming, Elizabeth A Spencer, Matthew J Thompson, \\ Richard J Stevens
}

To cite: Ashdown HF, Fleming S, Spencer EA, et al. Diagnostic accuracy study of three alcohol breathalysers marketed for sale to the public. BMJ Open 2014;4: e005811. doi:10.1136/ bmjopen-2014-005811

- Prepublication history and additional material is available. To view please visit the journal (http://dx.doi.org/ 10.1136/bmjopen-2014005811)

Received 30 May 2014 Revised 10 September 2014 Accepted 15 December 2014

CrossMark

Nuffield Department of Primary Care Health Sciences, University of Oxford, Oxford, UK

Correspondence to Dr Helen F Ashdown; helen. ashdown@phc.ox.ac.uk

\section{ABSTRACT}

Objectives: To assess the diagnostic accuracy of three personal breathalyser devices available for sale to the public marketed to test safety to drive after drinking alcohol.

Design: Prospective comparative diagnostic accuracy study comparing two single-use breathalysers and one digital multiuse breathalyser (index tests) to a police breathalyser (reference test).

Setting: Establishments licensed to serve alcohol in a UK city.

Participants: Of 222 participants recruited, 208 were included in the main analysis. Participants were eligible if they were 18 years old or over, had consumed alcohol and were not intending to drive within the following $6 \mathrm{~h}$.

Outcome measures: Sensitivity and specificity of the breathalysers for the detection of being at or over the UK legal driving limit $(35 \mu \mathrm{g} / 100 \mathrm{~mL}$ breath alcohol concentration).

Results: $18 \%$ of participants $(38 / 208)$ were at or over the UK driving limit according to the police breathalyser. The digital multiuse breathalyser had a sensitivity of $89.5 \%(95 \% \mathrm{Cl} 75.9 \%$ to $95.8 \%)$ and a specificity of $64.1 \%(95 \% \mathrm{CI} 56.6 \%$ to $71.0 \%)$. The single-use breathalysers had a sensitivity of $94.7 \%(95 \% \mathrm{Cl} 75.4 \%$ to $99.1 \%)$ and $26.3 \%$ $(95 \% \mathrm{Cl} 11.8 \%$ to $48.8 \%)$, and a specificity of $50.6 \%(95 \% \mathrm{Cl} 40.4 \%$ to $60.7 \%)$ and $97.5 \%$ (95\% Cl $91.4 \%$ to $99.3 \%$ ), respectively.

Self-reported alcohol consumption threshold of 5 UK units or fewer had a higher sensitivity than all personal breathalysers.

Conclusions: One alcohol breathalyser had sensitivity of $26 \%$, corresponding to false reassurance for approximately one person in four who is over the limit by the reference standard, at least on the evening of drinking alcohol. The other devices tested had $90 \%$ sensitivity or higher. All estimates were subject to uncertainty. There is no clearly defined minimum sensitivity for this safetycritical application. We conclude that current regulatory frameworks do not ensure high sensitivity for these devices marketed to consumers for a decision with potentially catastrophic consequences.

\section{Strengths and limitations of this study}

- Personal breathalysers are available for sale to the public in pharmacies for assessing safety to drive following consuming alcohol, some very inexpensively, and in some jurisdictions is now promoted by law.

- Accuracy of personal breathalyser devices has never previously been studied.

- This study tested diagnostic accuracy of these devices in a real-life setting including participant estimation of readings.

- Limitations include the uncontrolled environment of public houses and bars, use of a pragmatic reference standard, and wide $\mathrm{Cls}$ for some results due to low prevalence of those over the driving limit

- However, our conclusions for the worst performing device are robust even against this uncertainty, since even the upper confidence limit for sensitivity of the worst performing device $(48.8 \%)$ would still mean that about one in two individuals would be falsely reassured and this could lead to potentially dangerous driving decisions.

\section{INTRODUCTION}

Road traffic collisions (RTCs) are the second leading cause of death worldwide among people aged 5-29 years, estimated at 1.2 million deaths each year, and forecast to rise further by 2020. ${ }^{1}$ Consumption of alcohol is an important factor influencing the likelihood and severity of RTCs, and has been found to be a causal factor in $17-40 \%$ of RTCs worldwide. $^{2}$ The risk of an RTC increases rapidly with increasing blood alcohol concentration (BAC), with relative risk rising significantly beyond a BAC of $0.04 \mathrm{~g}$ alcohol per $100 \mathrm{~mL}$ blood $(\mathrm{g} / \mathrm{dL})$ to reach a relative risk of approximately 5 at $0.10 \mathrm{~g} / \mathrm{dL}^{2}{ }^{2}$ Introduction of maximum legal BAC limits for driving, which vary from 0.02 (eg, Russia) to $0.15 \mathrm{~g} / \mathrm{dL}$ (eg, Uganda), has been effective in reducing alcohol-related injuries and deaths. ${ }^{1}$ 
Alcohol breathalysers, which have long been available to law enforcement agencies, are now marketed direct to consumers, for example in some UK pharmacies and motoring stores, to test safety to drive following drinking alcohol, including the morning after. In July 2012, it became a legal requirement for drivers in France to carry a personal breathalyser at all times. ${ }^{3}$ Devices marketed to consumers are order of magnitudes lower in cost than those intended for law enforcement: for example at the time of writing the Alcosense Single is more than 300 times cheaper than the Dräger 6510 Home Office certified police breathalyser. ${ }^{4}$

The theoretical accuracy of breath alcohol measurement as a surrogate for blood alcohol measurement has been well-studied, ${ }^{6}$ and in the UK breath alcohol forms part of the prescribed legal limit, along with blood and urine alcohol concentration. ${ }^{7}$ However to the best of our knowledge the accuracy of devices currently marketed to the consumer/motorist has not been studied. Many such devices carry regulatory approvals such as the Conformité Européenne (CE), French NF certification and British Standard (BSI Kitemark) but in general such marks are statements of engineering quality rather than diagnostic accuracy. We therefore aimed to assess the diagnostic accuracy of personal breathalysers compared with a police breathalyser for detection of being at or over the UK legal driving limit in a real world situation representing a possible application for such devices.

\section{METHODS}

\section{Index tests and reference standard}

We selected for study as index tests the Alcosense Single, the UK counterpart of an NF-approved device widely sold for motorists in France; the Dräger Alco-Check, as a comparable single-use device from a competing manufacturer; and the Alcosense Elite, as an example of a digital multiuse device readily available from pharmacies, high street and online stores (Boots, Halfords, Amazon and others). We selected as reference standard the Dräger Alcotest 6510 device. This has Home Office approval and is standard issue to UK police for use as an initial test at the roadside, ${ }^{5}$ and is approved in the USA as an evidential breath-testing device (National Highway Traffic Safety Administration Standard 49 FR 48854) meaning readings can be used as evidence for prosecution of drink-driving offences in US courts. Manufacturer information states measurement precision as $\pm 0.008 \mathrm{mg} / \mathrm{L}$ or $1.7 \%$ of measurement value. ${ }^{8}$ The Dräger Alcotest 6510 and Alcosense Elite devices come with instruction manuals including details of breathalyser operating technique and result interpretation. The single-use devices contain information on their packaging and an enclosed sheet of paper including details of breathalyser operating technique and result interpretation. These manuals and leaflets recommended at least $15 \mathrm{~min}$ (Dräger Alcocheck), at least 20 min (Dräger 6510 and Alcosense Single), or at least $30 \mathrm{~min}$ (Alcosense Elite) elapse between alcohol consumption and use. All personal breathalyser instructions clearly state that any amount of alcohol even below the limit can impair driving ability.

\section{Study participants}

To test the breathalysers in a real world situation, representing one possible application for personal breathalysers, we recruited participants from establishments licensed to serve alcohol in the city centre of Oxford, UK, including college bars and public houses. In the absence of prior data with which to estimate sample size, we decided to recruit 200 participants, which would allow us to report the prevalence of intoxication with small SE (maximum 3.5\%). Recruitment took place on evenings during the period from October 2012 to January 2013: 11 study evenings were required to reach a sample size greater than 200. Participants were eligible if they were 18 years or over, had consumed alcohol, and were not intending to drive within the following $6 \mathrm{~h}$. We excluded potential drivers for ethical reasons.

\section{Recruitment and consent}

Individuals in these establishments were informally approached by a member of the research team and given preliminary information about the study. Potentially eligible interested participants were then asked to sit with the research team, at tables reserved within the same premises, where they received a full description of the study, were asked to read the study literature, and given an opportunity to ask further questions. Eligibility was checked and written consent taken. Participants were given a card with contact details to use in the event of withdrawing consent subsequently (eg, the next day): in the event, no participants used this option to withdraw consent.

\section{Study procedures}

The research team consisted of between two and four researchers, who followed the reference breathalyser manufacturers' written instructions in directing their use: a minimum of 20 min was enforced between recruitment and using the breathalyser devices, and participants were asked not to drink further alcohol, smoke, use mouthwash or drink fruit juice during this period. Participants were provided with water and asked to take at least one sip in order to clear any residual alcohol from the upper airway. During this $20 \mathrm{~min}$ period basic demographic details (age and sex) and reported alcohol consumption during the preceding $12 \mathrm{~h}$ were recorded by the researchers. Participants were not required to remain under observation during the 20 min period.

The 20 min waiting period did not comply with the manufacturers' instructions for one of the devices (Alcosense Elite). However, it was felt that a longer gap between recruitment and testing would result in high attrition rates, and increased potential for protocol violation by participants (eg, by smoking or drinking liquids other than water). We therefore compromised on a 
waiting time of $20 \mathrm{~min}$ based on the instructions for the reference device, and the fact that this complied with manufacturer instructions for the other two index devices.

Participants then used three breathalysers, at intervals of at least $1 \mathrm{~min}$, at the study tables under the supervision of a research team member. Each participant used, in random order, the reference standard, the Alcosense Elite multiuse and a randomly selected single-use breathalyser. Randomisation was carried out in advance by study number in random permuted blocks.

A member of the research team recorded digital outputs of the multiuse and reference breathalysers. The single-use breathalysers require subjective assessment of colour of crystals after use: we recorded assessments by researcher (primary outcome) and participant (secondary outcome). Participants were blinded to the researcher assessment and to the reference result.

\section{Statistical analysis}

We calculated sensitivity, specificity, positive and negative predictive values of the breathalysers for the outcome of being at or over the current UK legal driving $\operatorname{limit}^{7}$ of $35 \mu \mathrm{g} / 100 \mathrm{~mL}$ or $0.8 \%$ o BAC according to the reference standard. Self-reported alcohol consumption, converted to UK alcohol units ( 1 unit $=8 \mathrm{~g}$ alcohol) using nutritional tables, ${ }^{9}$ was also assessed for diagnostic accuracy of being at or over the UK legal driving limit, and a receiver operating characteristic (ROC) analysis undertaken to assess different unit thresholds. Statistical calculations were carried out using standard methods ${ }^{10}$ and the ROC analysis using Stata (Release V.11). Minor

Figure 1 Study flow chart. All participants (except exclusions detailed in figure) were tested with the Dräger Alcotest $6510(\mathrm{R})$, the Alcosense Elite $\left(I_{M}\right)$, and one of either the Dräger Alco-Check $\left(I_{S D}\right)$ or the Alcosense Single $\left(\mathrm{I}_{\mathrm{SA}}\right)$. The order of undertaking each breathalyser, and the selection of $I_{S D}$ or $I_{S A}$ was determined by randomisation. ${ }^{*}$ Three participants left before analysis therefore results for participant estimation of $I_{S A}$ are only available for 97 participants.

protocol violations were discussed among the research team, with sensitivity analyses performed to determine whether there was any difference in overall results due to inclusion or exclusion of these. Participants with missing data (eg, sex, self-reported alcohol consumption or participant estimation of result) were included except for analyses involving the missing component itself.

\section{RESULTS}

A total of 208 participants were included in the main analysis (see figure 1), of whom 148/207 (71.5\%) were male and with a median age of 20. Participants reported having consumed a median of 6 UK units of alcohol (range 1-25), equivalent to a median of $46 \mathrm{~g}$ (range 8-204) alcohol. One hundred and eight participants were tested with the Dräger Alco-Check single-use breathalyser, and 100 with the Alcosense Single singleuse breathalyser.

Thirty-eight participants $(18.3 \%, 95 \%$ CI $11.7 \%$ to $27.4 \%$ ) were at or over the current UK driving limit of $35 \mu \mathrm{g} / 100 \mathrm{~mL}$ according to the reference breathalyser. Table 1 compares performance of the three index breathalysers at detecting those at or over the UK limit. Compared with the reference breathalyser, the Alcosense Elite multiuse breathalyser had a sensitivity of $89.5 \%$ (95\% CI $75.9 \%$ to $95.8 \%)$, the Dräger Alco-Check single-use breathalyser had a sensitivity of 94.7\% (95\% CI $75.4 \%$ to $99.1 \%$ ) and the Alcosense Single breathalyser had a sensitivity of $26.3 \%$ (95\% CI $11.8 \%$ to $48.8 \%$ ). When analyses were repeated using the participant's interpretation of colour change in the

222 recruited

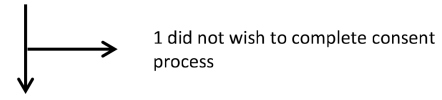

221 consented

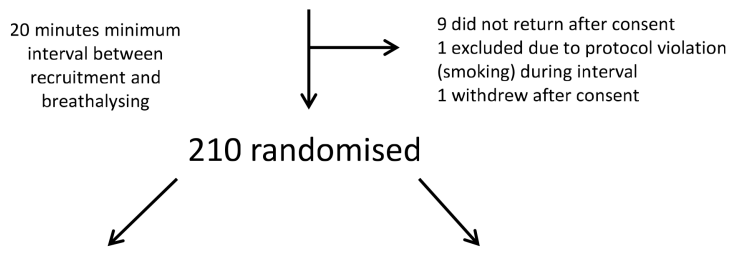

108 randomised to

$\mathrm{R}, \mathrm{I}_{\mathrm{M}}, \mathrm{I}_{\mathrm{SD}}$ in random order

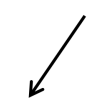

105 completed $R, I_{M}, I_{S D}$

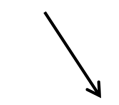

3 completed $\mathrm{R}$ and $\mathrm{I}_{\mathrm{SD}}$ only due to device $I_{M}$ error $\geq 3$ attempts
102 randomised to

$\mathrm{R}, \mathrm{I}_{\mathrm{M}}, \mathrm{I}_{\mathrm{SA}}$ in random order

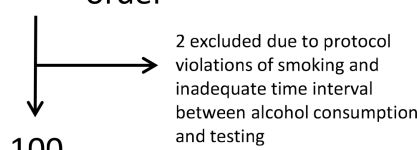

100 completed

$\mathrm{R}, \mathrm{I}_{\mathrm{M}}, \mathrm{I}_{\mathrm{SA}}$ * 


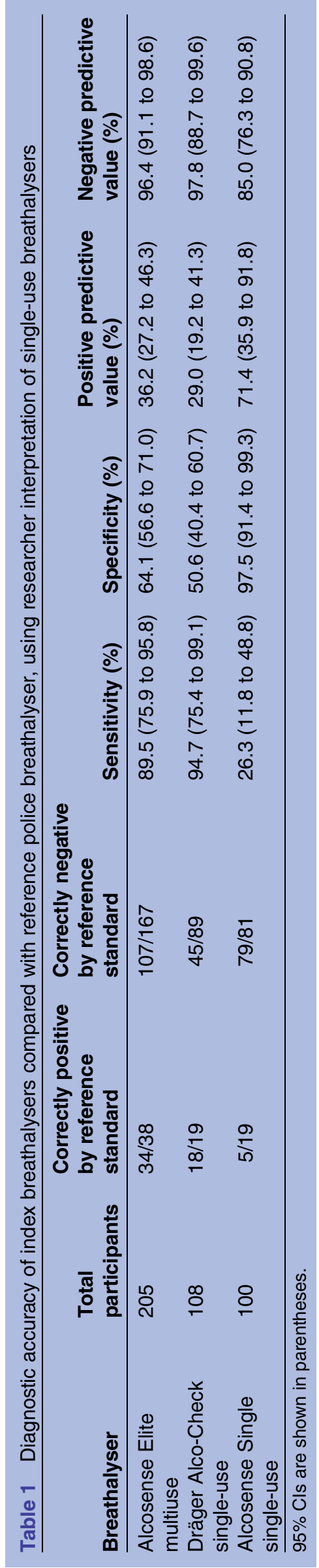

single-use breathalysers instead of researcher's interpretation, sensitivity was $94.7 \%$ (95\% CI $75.4 \%$ to $99.1 \%$ ) for the Dräger Alco-Check (ie, identical to researcher estimation) and $16.7 \%$ (95\% CI $11.8 \%$ to $48.8 \%$ ) for the Alcosense Single (see online supplementary appendix 1 for full data for participant estimation).

We conducted three sensitivity analyses in turn (1) excluding two results where the participants were suspected to have incorrectly used the device, (2) a colourblind participant who may have had difficulty interpreting the colour change of crystals and (3) a participant who was suspected to have violated the protocol (consumed alcohol between use of the three devices). In general, these analyses showed minimal difference to results overall (see online supplementary appendix 1) with the exception that the sensitivity of the Dräger Alco-Check increased to $100 \%$ (95\% CI $83.2 \%$ to $100 \%$ ) in the latter sensitivity analysis. There were no reported adverse events from using any of the breathalyser devices.

Because there is no single standard threshold for driving decisions based on self-estimated alcohol consumption, we calculated sensitivity and specificity for all alcohol unit thresholds (table 2) and plotted them in ROC space (figure 2).

\section{DISCUSSION}

We have shown that breathalysers available for sale to the public for personal use vary considerably in their performance in detecting being at or over a legal driving limit during the period directly after drinking. Two of the devices tested, the Alcosense Elite digital multiuse breathalyser and Dräger Alco-Check single-use breathalyser had a sensitivity of approximately $90 \%$ and $95 \%$, respectively, in the main analyses. However, even a sensitivity of $95 \%$ means that approximately 1 in 20 people over the driving limit would be falsely reassured by these tests. We question whether even this would be sufficient sensitivity to assess safety to drive. The third device, the Alcosense Single single-use breathalyser, had a sensitivity of only $26 \%$, meaning that only approximately one in four individuals over the legal limit would be identified by this device. Participants (rather than researchers) interpreting results, which is what would occur in real life, reduced sensitivity further to only $17 \%$. This device has a correspondingly high specificity, but specificity is not the safety-critical aspect of performance of a device assessing safety to drive. Surprisingly, we found that self-reported consumption of alcohol was a more sensitive test for being at or over the legal driving limit than the three breathalysers tested for up to 5 UK units of alcohol consumed. None of the devices outperformed simple recall of amount of alcohol consumed up to $5 \mathrm{UK}$ units of alcohol.

Strengths of our study include testing participants in a 'real world' environment including assessment of participants' estimation of breathalyser readings, which enables 
Table 2 Diagnostic accuracy of self-reported alcohol consumption compared with reference police breathalyser

\begin{tabular}{|c|c|c|c|c|c|}
\hline $\begin{array}{l}\text { Threshold of } \\
\text { self-reported alcohol } \\
\text { consumption } \\
\text { (UK units) }\end{array}$ & $\begin{array}{l}\text { Total } \\
\text { participants }\end{array}$ & $\begin{array}{l}\text { Correctly } \\
\text { positive by } \\
\text { reference } \\
\text { standard }\end{array}$ & $\begin{array}{l}\text { Correctly } \\
\text { negative by } \\
\text { reference } \\
\text { standard }\end{array}$ & Sensitivity (\%) & Specificity (\%) \\
\hline 1 & 207 & $38 / 38$ & 9/169 & $100.0(90.8$ to 100.0$)$ & 5.3 (2.8 to 9.8$)$ \\
\hline 2 & 207 & $38 / 38$ & 23/169 & $100.0(90.8$ to 100.0$)$ & 13.6 (9.2 to 19.6$)$ \\
\hline 3 & 207 & $38 / 38$ & $52 / 169$ & $100.0(90.8$ to 100.0$)$ & 30.8 (24.3 to 38.1$)$ \\
\hline 4 & 207 & $38 / 38$ & $71 / 169$ & $100.0(90.8$ to 100.0$)$ & 42.0 (34.8 to 49.5$)$ \\
\hline 5 & 207 & $37 / 38$ & $84 / 169$ & 97.4 (86.5 to 99.5$)$ & 49.7 (42.3 to 57.2$)$ \\
\hline 6 & 207 & $35 / 38$ & $105 / 169$ & 92.1 (79.2 to 97.3$)$ & 62.1 (54.6 to 69.1 ) \\
\hline 7 & 207 & $34 / 38$ & $117 / 169$ & 89.5 (75.9 to 95.8$)$ & 69.2 (61.9 to 75.7$)$ \\
\hline 8 & 207 & $29 / 38$ & $130 / 169$ & 76.3 (60.8 to 87.0$)$ & 76.9 (70.0 to 82.6$)$ \\
\hline 9 & 207 & $26 / 38$ & $142 / 169$ & 68.4 (52.5 to 80.9$)$ & 84.0 (77.7 to 88.8$)$ \\
\hline 10 & 207 & $20 / 38$ & $148 / 169$ & 52.6 (37.3 to 67.5$)$ & 87.6 (81.8 to 91.7$)$ \\
\hline
\end{tabular}

$1 \mathrm{UK}$ unit is equivalent to $10 \mathrm{~mL}$ or $8 \mathrm{~g}$ alcohol (total 207 participants due to missing data on alcohol consumption for 1 participant). $95 \%$ Cls are shown in parentheses.

generalisability to everyday life. However, this pragmatic approach also brings limitations in that the setting in college bars and public houses could not be rigorously controlled. It is possible, for example, that poor lighting, or unknown protocol violation by participants who could not always be perfectly monitored in this busy environment, could have introduced some inaccuracies. Operating the three breathalysers in close succession and randomisation of order of use of the breathalysers should have helped reduce the risk of such effects causing bias in the overall results. There is also the possibility that ambient alcohol vapour in the environment may have resulted in excess false positives. As discussed above, we may have underestimated the sensitivity of the Dräger Alco-Check because of a suspected protocol violation. Three participants inadvertently took the breathalysers in a different order to that planned: we decided to

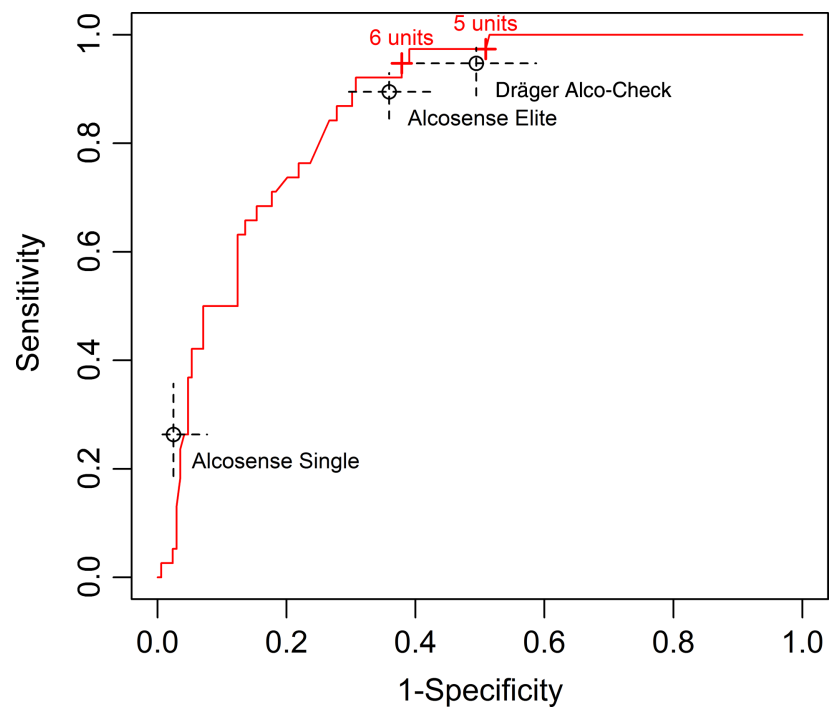

Figure 2 Receiver operating characteristic curve of self-reported alcohol consumption in UK units, with comparative sensitivity and specificity for breathalysers tested. include these participants because the aim was to have an overall variety in breathalyser order and this was unlikely to have any impact for such a small number of participants. The use of a $20 \mathrm{~min}$ rather than $30 \mathrm{~min}$ minimum time after drinking alcohol was not adherent to the manufacturer's instructions for the Alcosense Elite, and so it is possible that this may have affected results for this breathalyser. Hyperventilation immediately prior to a breath sample reduces breath alcohol concentration ${ }^{11}$ and breath holding increases it. ${ }^{12}{ }^{13} \mathrm{We}$ recorded a minimum amount of information about participants to facilitate recruitment and monitoring breathing would not have been possible in this deliberately real-life environment. However, we followed the instructions in the Alcosense Elite manual to "wait until you are breathing normally again" by ensuring that participants were relaxed and ensuring at least a minute between breathalysers for any recovery required. Participants were typically seated while completing the consent process and initial data collection before breathalysing took place. Randomising the order of breathalysing would further reduce any bias in the overall results due to temporal effects such as hyperventilation before the first test. The high solubility of alcohol means that it is thought to be deposited in exhaled air largely from the proximal airway conducting system, and breath alcohol concentration is not therefore reliant on alveolar equilibration. ${ }^{14}$ Our methodology of allowing $1 \mathrm{~min}$ between each test was designed to allow satisfactory measurement of breath alcohol concentration.

Participants were blinded to their result on the reference breathalyser until after completing estimation of colour change, however researchers were not and it is possible that this could have introduced some bias. We met our planned recruitment target, but the prevalence of those over the legal limit by the reference standard means that our CIs are fairly wide. Even using the upper confidence limit for sensitivity, however, the worstperforming breathalyser would still have sensitivity 
under $50 \%$. Our sample of participants obtained from colleges and pubs may not be representative of the population who purchase personal breathalysers, particularly in age group and quantity of alcohol consumed and sociodemographics; although this might affect sensitivity of self-reported alcohol consumption, it should not impact significantly on the overall accuracy of the breathalyser devices. We tested the index devices on the night of drinking and in a bar environment and the generalisability to other uses such as 'morning-after' use may be unclear; blood and breath alcohol concentrations decline with time after drinking alcohol at similar rates and maintain high correlation, ${ }^{15}$ but it is possible that diagnostic accuracy of personal breathalysers may differ at lower alcohol concentrations.

Use of blood alcohol as a gold standard was not possible in this pragmatic, field study. We chose as our reference standard the Dräger Alcotest 6510 which has passed a Home Office testing protocol requiring error less than $10 \%$ in all readings ${ }^{16}$ and is also an evidential device for criminal prosecutions in the USA. The devices currently approved for evidential use in the UK are not portable and were therefore not suitable for our study setting. For convenience we selected index devices easily available in the UK for testing, however the same devices calibrated for different legal limits are sold outside of the UK, and other devices sold elsewhere use similar technology and are similarly priced. Therefore, while we cannot directly apply our results to other countries, we would anticipate similar findings elsewhere.

We have not found other studies testing the accuracy of personal breathalysers. Studies in the USA of college student parties have found a mean BAC of $0.077 \%$ (SD $0.063 \%$ ) (for context, the UK driving limit is $0.08 \%$ ). ${ }^{18}$ A Canadian study found a linear relationship between self-reported alcohol consumption and BAC in emergency room attendees, up to seven drinks. ${ }^{19}$ However, a US study of college students, which compared estimated BAC to measured BAC, found that students tended to over-estimate their levels of consumption when surveyed in the midst of a night of drinking. ${ }^{20}$ We did not attempt to convert self-reported alcohol consumption to BAC and only recorded total quantity consumed, which would have had differential effects between individuals dependent on weight, sex and the time over which the drinks were consumed. However, despite the variation these factors would introduce into BAC, self-reported consumption up to 5 units was still a more sensitive test than the breathalysers tested, and BAC is known to correlate poorly with symptoms of intoxication. ${ }^{21}$

Our research suggests that at least some personal breathalysers available for sale to the public are not always sufficiently sensitive to test safety to drive after drinking alcohol, where use of inaccurate information from breathalysers thought to be accurate could have catastrophic safety implications for drivers. The fact that these devices are sold in well-established pharmacies including national chains does not guarantee sufficient accuracy for safe use. Medical and measurement devices may carry regulatory approvals such as CE or NF marking, but this does not appear to correlate with accuracy, and this raises wider questions over how this marking may be perceived by users. A derivative device of the worst-performing breathalyser in our study is widely sold for use in France as part of the new law requiring breathalysers to be carried when driving, and has French NF approval. ${ }^{4}$ Although results from our study cannot be directly applied to the lower French driving limit of $0.05 \mathrm{~g} / \mathrm{dL}$ and a derivative device, they question the utility of the new law which on the one hand may improve public awareness of drink-driving in general, but risks ill-informed driving decisions based on inaccurate results from a personal breathalyser. Replication of our results in other settings and with other breathalysers could further inform policymakers planning to introduce similar laws in other jurisdictions, and explore the characteristics of the population who purchase personal breathalysers and how they use the results obtained. Finally, our research raises worrying questions about the level of scrutiny that medical tests intended for sale to the public undergo in Europe, and raises wider concerns about how diagnostic accuracy in particular is evaluated, and whether any further field evaluations are required for intended users, perceptions of accuracy of such devices and how use of such devices interacts with medical testing in other healthcare settings.

Acknowledgements The authors would like to thank the study participants, and those colleges of the University of Oxford and public houses in Oxford which permitted them to recruit on their premises; Michael Ashdown for assistance with legal literature; and David McCartney for additional advice on the final manuscript. They would also like to thank the reviewers of this article for their hard work and insightful comments.

Contributors All authors contributed to study design, data collection, data interpretation and helped write the paper. RJS conceived the idea for the study. HFA, EAS and RJS analysed the data. HFA is the guarantor. All authors approved the final version of the manuscript.

Disclaimer The views expressed in this article are those of the authors and not necessarily those of the NHS, the NIHR or the Department of Health.

Funding This study received no external funding.

Competing interests None.

Ethics approval The study was approved by the University of Oxford Medical Sciences Division Interdivisional Research Ethics Committee (reference MSD/ IDREC/2011/13).

Patient consent All participants gave informed consent before taking part.

Provenance and peer review Not commissioned; externally peer reviewed.

Data sharing statement All authors had full access to all of the data (including statistical reports and tables) in the study and can take responsibility for the integrity of the data and the accuracy of the data analysis. Full data are available from the corresponding author on request. Consent for data sharing was not obtained but the presented data are anonymised and risk of identification is low.

Open Access This is an Open Access article distributed in accordance with the Creative Commons Attribution Non Commercial (CC BY-NC 4.0) license, which permits others to distribute, remix, adapt, build upon this work noncommercially, and license their derivative works on different terms, provided 
the original work is properly cited and the use is non-commercial. See: http:// creativecommons.org/licenses/by-nc/4.0/

\section{REFERENCES}

1. Peden M, Scurfield R, Sleet $\mathrm{D}$, et al. World report on road traffic injury prevention. Geneva: World Health Organization, 2004.

2. Global Road Safety Partnership. Drinking and driving: a road safety manual for decision-makers and practitioners. Geneva: World Health Organization, 2007.

3. Légifrance: Le Service Public pour la Diffusion du Droit: Décret $\mathrm{n}^{\circ}$ 2012-284 du 28 février 2012 relatif à la possession obligatoire d'un éthylotest par le conducteur d'un véhicule terrestre à moteur. http:// www.legifrance.gouv.fr/affichTexte.do?cidTexte=JORFTEXT 000025417826\&categorieLien=id (accessed May 2014).

4. Halfords store website. Alcosense Single breathalyser. http://www. halfords.com/webapp/wcs/stores/servlet/product_storeld_10001 catalogld_10151_productld_871069_langld_-1_categoryld_226211 (accessed May 2014).

5. UK Breathalysers website. Draeger Police 6510. http://www. ukbreathalysers.com/2_alcohol/test_others/prob/6510.html (accessed May 2014).

6. Gibb KA, Yee AS, Johnston CC, et al. Accuracy and usefulness of a breath alcohol analyzer. Ann Emerg Med 1984;13:516-20.

7. Road Traffic Act 1988 Chapter 52 Part I Sections 4-11. Motor vehicles: drink and drugs. Ministry of Justice. http://www.legislation. gov.uk/ukpga/1988/52/part///crossheading/ motor-vehicles-drink-and-drugs (accessed May 2014).

8. Dräger Alcotest 6510 product specification 2012.

9. McCance RA, Widdowson EM. McCance and Widdowson's The Composition of Foods. 6th edition compiled by the Food Standards Agency and Institute of Food Research. Cambridge, UK: Royal Society of Chemistry, 2002.

10. Newcombe RG, Altman DG. Proportions and their differences. In: Altman DG, Bryant TN, Gardner MJ, eds. Statistics with confidence. 2nd edn. London: BMJ Books, 2000:46-54.
11. Ohlsson J, Ralph DD, Mandelkorn MA, et al. Accurate measurement of blood alcohol concentration with isothermal rebreathing. J Stud Alcohol 1990;51:6-13.

12. Jones AW. How breathing technique can influence the results of breath-alcohol analysis. Med Sci Law 1982;22:275-80.

13. Jones AW. Role of rebreathing in determination of the blood-breath ratio of expired ethanol. J Appl Physiol Respir Environ Exerc Physiol 1983;4:1237-41.

14. Hlastala MP. The alcohol breath test-a review. J Appl Physiol 1998;84:401-8.

15. Jones AW, Andersson L. Comparison of ethanol concentrations in venous blood and end-expired breath during a controlled drinking study. Forensic Sci Int 2003;132:18-25.

16. Parliamentary Under-Secretary of State, Home Office. The Preliminary Breath Test Device Approval 2005. https://www.gov.uk/ government/uploads/system/uploads/attachment_data/file/117005/ draeger-lion-envitec-alcoquant.pdf (accessed Aug 2014).

17. Home Office and Forensic Science Service. Breath Alcohol Screening Devices: a guide to type-approval procedures for breath alcohol screening devices used for law enforcement in Great Britain (Version 1.0, 30/06/2004). https://www.gov.uk/ government/uploads/system/uploads/attachment_data/file/ 117043/breathalcoholscreening.pdf (accessed Aug 2014).

18. Clapp JD, Min JW, Shillington AM, et al. Person and environment predictors of blood alcohol concentrations: a multi-level study of college parties. Alcohol Clin Exp Res 2008;32:100-7.

19. Bond J, Ye Y, Cherpitel CJ, et al. The relationship between self-reported drinking and BAC level in emergency room injury cases: is it a straight line? Alcohol Clin Exp Res 2010;34:1118-25.

20. Kraus CL, Salazar NC, Mitchell JR, et al. Inconsistencies between actual and estimated blood alcohol concentrations in a field study of college students: do students really know how much they drink? Alcohol Clin Exp Res 2005;29:1672-6.

21. Olson KN, Smith SW, Kloss JS, et al. Relationship between blood alcohol concentration and observable symptoms of intoxication in patients presenting to an emergency department. Alcohol Alcohol 2013;48:386-9. 
Correction: Diagnostic accuracy study of three alcohol

breathalysers marketed for sale to the public

Ashdown HF, Fleming S, Spencer EA, et al. Diagnostic accuracy study of three alcohol breathalysers marketed for sale to the public. BMJ Open 2014;4:e005811. doi: 10.1136/ bmjopen-2014-005811

There is an error in the following sentence:

"One alcohol breathalyser had sensitivity of $26 \%$, corresponding to false reassurance for approximately one person in four who is over the limit by the reference standard, at least on the evening of drinking alcohol".

The part 'one person in four who is' should read 'three people in four who are'.

Open Access This is an Open Access article distributed in accordance with the Creative Commons Attribution Non Commercial (CC BY-NC 4.0) license, which permits others to distribute, remix, adapt, build upon this work non-commercially, and license their derivative works on different terms, provided the original work is properly cited and the use is non-commercial. See: http://creativecommons.org/licenses/by-nc/4.0/

C Article author(s) (or their employer(s) unless otherwise stated in the text of the article) 2018. All rights reserved. No commercial use is permitted unless otherwise expressly granted.

BMJ Open 2018;8:e005811corr1. doi:10.1136/bmjopen-2014-005811corr1

A) Check for updates 